

\title{
A Nano-Dose Protocol For Cobb Angle Assessment in Children With Scoliosis: Results of a Phantom-based and Clinically Validated Study
}

Peter H. Pedersen, Claudio Vergari, Alexia Tran, Fred Xavier, Antoine Jaeger, Pierre Laboudie, Victor Housset, Soren Eiskjaer, Raphaël Vialle

\section{To cite this version:}

Peter H. Pedersen, Claudio Vergari, Alexia Tran, Fred Xavier, Antoine Jaeger, et al.. A NanoDose Protocol For Cobb Angle Assessment in Children With Scoliosis: Results of a Phantombased and Clinically Validated Study. Clinical Spine Surgery, 2019, 32 (7), pp.340-345. 10.1097/BSD.0000000000000834 . hal-02529647

\section{HAL Id: hal-02529647 \\ https://hal.science/hal-02529647}

Submitted on 2 Apr 2020

HAL is a multi-disciplinary open access archive for the deposit and dissemination of scientific research documents, whether they are published or not. The documents may come from teaching and research institutions in France or abroad, or from public or private research centers.
L'archive ouverte pluridisciplinaire HAL, est destinée au dépôt et à la diffusion de documents scientifiques de niveau recherche, publiés ou non, émanant des établissements d'enseignement et de recherche français ou étrangers, des laboratoires publics ou privés. 


\title{
A Nano-Dose Protocol For Cobb Angle Assessment in Children With Scoliosis: Results of a Phantom-based and Clinically Validated Study
}

\author{
Peter H. Pedersen, MD, ${ }^{*}$ Claudio Vergari, PhD, $\dagger$ Alexia Tran, MD, $\neq$ Fred Xavier, MD, PhD, $\S$ \\ Antoine Jaeger, MD, || Pierre Laboudie, MD,\| Victor Housset, MD, || Søren P. Eiskjar, MD, * \\ and Raphäel Vialle, $M D, P h D \|$
}

Study design: This was a prospective validation study with technical notes.

Objective: This study aimed to validate a new ultra-low-dose full-spine protocol for reproducible Cobb angle measurementsthe "nano-dose" protocol.

Summary of Background Data: Scoliosis is a 3-dimensional (3D) deformity of the spine characterized by $3 \mathrm{D}$ clinical parameters. Nevertheless, 2D Cobb angle remains an essential and widely used radiologic measure in clinical practice. Repeated imaging is required for the assessment and follow-up of scoliosis patients. The resultant high dose of absorbed radiation increases the potential risk of developing radiation-induced cancer in such patients. Micro-dose radiographic imaging is already available in clinical practice, but the radiation dose delivered to the patient could be further reduced.

Methods: An anthropomorphic child phantom was used to establish an ultra-low-dose protocol in the EOS Imaging System still allowing Cobb angle measurements, defined as nano-dose. A group of 23 consecutive children presenting for scoliosis assessment, 12 years of age or younger, were assessed with standarddose or micro-dose and additional nano-dose full-spine imaging modalities. Intraobserver and interobserver reliability of determining the reliability of $2 \mathrm{D}$ Cobb angle measurements was performed. The dosimetry was performed in the anthropomorphic phantom to confirm theoretical radiation dose reduction.

Results: A nano-dose protocol was established for reliable Cobb angle measurements. Dose area product with this new nano-dose

Received for publication August 21, 2018; accepted April 19, 2019.

From the *Department of Orthopedic Surgery, University Hospital of Aalborg, Aalborg, Denmark; †Arts et Metiers ParisTech, LBM/ Institut de Biomecanique Humaine Georges Charpak; tDepartment of Radiology, Armand Trousseau Hospital, Sorbonne Université, APHP Paris, Paris, France; §Department Surgery (Spine), QEII Health Sciences Centre-Halifax Infirmary, Dalhousie University, Halifax, Canada; and \|Department of Pediatric Orthopedics, Armand Trousseau Hospital, Sorbonne Université, APHP Paris, Paris, France.

R.V. has received honoraria from EOS Imaging, Paris, France. The remaining authors declare no conflict of interest.

Reprints: Peter H. Pedersen, MD, Department of Orthopedic Surgery, University Hospital of Aalborg, Hobrovej 18-22, 9000 Aalborg, Denmark (e-mail: php@rn.dk). protocol was reduced to $5 \mathrm{mGy} \times \mathrm{cm}^{2}$, corresponding to one sixth of the micro-dose protocol $\left(30 \mathrm{mGy} \times \mathrm{cm}^{2}\right)$ and $<1 / 40$ th of the standard-dose protocol $\left(222 \mathrm{mGy} \times \mathrm{cm}^{2}\right)$. Theoretical dose reduction, for posteroanterolateral positioning was confirmed using phantom dosimetry. Our study showed good reliability and repeatability between the 2 groups. Cobb variability was $<5$ degrees from the mean using $95 \%$ confidence intervals.

Conclusions: We propose a new clinically validated nano-dose protocol for routine follow-up of scoliosis patients before surgery, keeping the radiation dose at a bare minimum, while allowing for reproducible Cobb angle measurements.

Key Words: Cobb angle, scoliosis, low dose imaging, ionizing radiation, repeatability

(Clin Spine Surg 2019;32:E340-E345)

S coliosis is a 3-dimensional (3D) deformity of the spine $S$ that can be characterized by $3 \mathrm{D}$ clinical parameters. The quantitative evaluation of scoliosis is important to follow its progression, and, when planning for conservative or surgical procedures, results in numerous radiologic examinations. High levels of cumulated absorbed radiation dose have been associated with an increased risk of cancer among these patients. ${ }^{1-3}$ To reduce this potential danger from iatrogenic ionizing radiation, considerable research into radiation dose optimization has been ongoing. The principal aim is to keep the radiation dose at a minimum while ensuring sufficient image quality for diagnosis and treatment, as per the ALARA principle (As Low As Reasonably Achievable). ${ }^{4,5}$ Various systems adhering to the ALARA principle exist; one such system is the EOS slot-scanner (EOS Imaging, Paris, France) ${ }^{6}$ The EOS system yields high-quality images at radiation doses lower than conventional x-ray systems, which has been described in detail previously. ${ }^{7-11}$ EOS standard-dose and micro-dose options are already available in clinical practice, but we hypothesized that the radiation delivered to the patient could be even further reduced, still allowing reproducible $\mathrm{Cobb}$ angle measurements.

The Cobb angle is the angle between the upper endplate and the lower endplate of the 2 vertebrae at the extremes of the assessed curvature. It is used to distinguish 
between normal and pathologic conditions of the spine. Cobb angle can be reliably measured from a 3D reconstruction of the spine. ${ }^{12}$ This method allows taking into account the patient's orientation relative to the imaging plane and the $3 \mathrm{D} / 2 \mathrm{D}$ projection bias. ${ }^{13}$ Nevertheless, 3D imaging capabilities in weight-bearing position are not yet widespread, and 2D Cobb angle remains an important and widely used radiologic measure in clinical practice. $2 \mathrm{D}$ Cobb angle is measured manually on a coronal $\mathrm{x}$-ray by drawing intersecting perpendicular lines to the above-mentioned endplates or, more commonly, by fixing landmark points parallel to these endplates in a picture archiving and communication system (PACS), automatically calculating the Cobb angle.

Differences between computer-assisted methods and manual methods have been evaluated in several review papers with regard to variability and reproducibility. ${ }^{14-17}$ The consensus states that a change of 5 degrees in Cobb angle over a 6-month period is consistent with a significant progression of spinal curvature. Moreover, the arbitrary limit between a "healthy" spine and a scoliotic spine is 10 degrees in the coronal plane. ${ }^{17}$ This study aimed to determine the lowest irradiation dose (nano-dose) that would still yield a reproducible 2D Cobb angle measurement, assuming a 5-degree uncertainty as acceptable.

\section{METHODS}

First, a phantom-based study was performed to semiquantitatively define the minimal dose allowing visibility of the spine, defined as "nano-dose," as detailed below. Then, a prospective cohort of scoliosis patients was recruited to determine the reliability of $2 \mathrm{D}$ Cobb angle measurement with nano-dose, which was compared with the reliability obtained on the same patients with subsequent micro-dose imaging.

\section{Establishing and Verifying Nano-Dose}

Initial steps to determine the lowest possible dose, still allowing Cobb angle measurements, were undertaken on an anthropomorphic phantom representing a 5-yearold child (ATOM-CIRS; Computerized Imaging Reference System Inc., Norfolk, VA). ${ }^{18}$ Successive reductions of scan parameters were followed by phantom imaging in posteroanterolateral (PAL) exposure. This technique is a common protocol at many institutions and is also the standard for conventional radiology of the spine. Doses were lowered by reducing the current $(\mathrm{mA})$ and the scan speed, which are both directly proportional to the radiation dose. For instance, halving the current leads to a $50 \%$ diminution in the radiation dose, and an increase from scan speed 3 to scan speed 2 further reduces the radiation dose by one third.

An experienced surgeon, blinded to the imaging settings, rated the images' quality on a scale from 1 to 5 . A sharp decrease in image quality was detected at dose area product (DAP) $<5 \mathrm{mGy} \times \mathrm{cm}^{2}$ for the coronal plane (Table 1), which was retained as the target nano-dose. Clinical pilot imaging was performed on pediatric patients being assessed for suspected scoliosis, at 3 different
TABLE 1. EOS Scan Parameters For Nano-Dose and MicroDose Protocols

\begin{tabular}{lccc}
\hline Acquisition Protocol & Nano-Dose & Micro-Dose & Standard-Dose \\
\hline Morphotype & Small & Small & Small \\
Scan speed & 2 & 3 & 4 \\
Coronal plane* & & & \\
$\quad$ kV & 60 & 60 & 83 \\
mA & 20 & 80 & 200 \\
DAP $\dagger\left(m G y \times \mathrm{cm}^{2}\right)$ & 5 & 30 & 222 \\
Lateral plane & & & \\
kV & 80 & 80 & 103 \\
mA & 20 & 80 & 200 \\
DAP $\dagger\left(\mathrm{mGy} \times \mathrm{cm}^{2}\right)$ & 11 & 67 & 371 \\
\hline
\end{tabular}

*Coronal posteroanterior positioning.

$\dagger$ Dose area product (DAP) (the absorbed dose multiplied by area irradiated) displayed for the pediatric phantom with a scan field height of $72 \mathrm{~cm}$.

settings around the previously selected nano-dose, to qualitatively evaluate image quality and confirm the nanodose of $5 \mathrm{mGy} \times \mathrm{cm}^{2}$. Table 1 shows the scan parameters for the chosen nano-dose setting and resulting reduction of DAP when applied to the phantom.

Dosimetry with thermoluminescent dosimeters was performed in the pediatric phantom with the chosen nanodose setting to determine the absorbed radiation dose. A method previously published by Damet et al ${ }^{10}$ was used to calculate effective dose from measured mean organ doses multiplied by organ-specific tissue weighting factors according to The International Committee on Radiological Protection (ICRP), publication 103. ${ }^{4}$

\section{Inclusions}

A consecutive group of 24 children going for routine full-spine imaging control of scoliosis were offered nanodose imaging in addition to micro-dose imaging instead of routine standard-dose imaging. A predefined age limit of 12 years of age was chosen. They did not suffer from any neurological or syndromic conditions, and they were able to stand by themselves without means of external help. Patients and parents were provided with written and oral information concerning the study, and signed consent was obtained for each patient. Our local ethics committee approved the study design and protocol. The 2 sets of images were taken one after the other within 2 minutes' time. The patients were carefully instructed to remain in the same positions during acquisitions.

All the patients were included from a juvenile or early-onset idiopathic scoliosis screening or follow-up examination.

\section{Reproducibility Assessment}

Five observers took part in the study, 4 orthopedic surgeons and 1 radiologist, all with experience of measuring Cobb angles. Curves were measured at predesignated levels to make sure that the same curves were measured. The same software and technical conditions were used (Carestream PACS Carestream Health Inc., Rochester, NY). As shown in Figure 1, Cobb angle was automatically calculated when digitizing 2 segments 

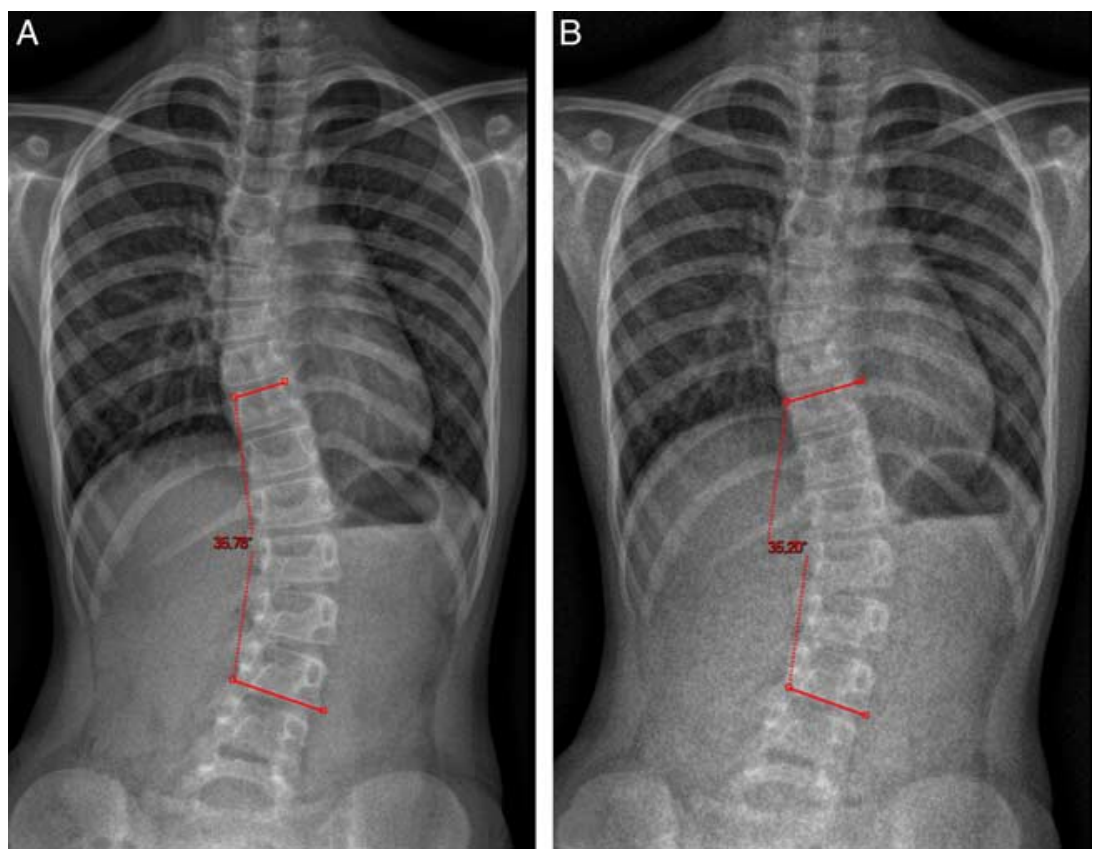

FIGURE 1. Cobb angle measurement with micro-dose $(A)$ and Cobb angle measurement with nano-dose (B).

parallel to endplates of the vertebra at each extreme of the curve. Observers rated micro-dose and nano-dose images 3 times each in a randomized order and over a 2-week period to avoid bias.

For those patients with a 2D Cobb angle $>10$ degrees, intraobserver and interobserver reliability of Cobb angle measurements were assessed, according to the ISO5725 standard, in terms of twice the SD of repeated measurements. For both radiation doses, Cobb angle limits of agreement were reported in Bland-Altman plots. Some of the patients who were imaged for the first suspicion of scoliosis, had Cobb angles < 10 degrees; for these patients, the reliability of the diagnosis was estimated as the number of Cobb angle measurements being higher or lower than 10 degrees.

\section{RESULTS}

A total of 23 children with a mean age of 11 years (range, 9-12 y) underwent micro-dose and nano-dose fullspine examinations. Two patients were excluded because of artifacts due to metallic implants close to the spine, which made image quality for nano-dose unacceptable, and no further patients with implants were included. One patient was excluded before nano-dose imaging owing to severe obesity [body mass index $(\mathrm{BMI}>30)$ ] rendering images with the micro-dose protocol of poor quality. A total of 630 Cobb angle measurements were made, 3 measurements per observer of 2 dose levels for each of the 21 patients.

Seven patients showed an average Cobb angle $<10$ degrees. For these patients, $88 \%$ of the Cobb angle measurements in the micro-dose (ie, 92 measurements of 105 ) were consistently $<10$ degrees. With nano-dose, $96 \%$ of the measurements (ie, 101 of 105) were $<10$ degrees.
For the remaining 14 patients with a Cobb angle $>10$ degrees, mean Cobb angle was 25 degrees (range, 11-49 degrees).

The intraobserver repeatability and interobserver reproducibility relative to the 2 dose levels are shown in Table 2. As expected, intraobserver repeatability was higher than interobserver reproducibility. The results showed good reliability, that is, $\leq 5$ degrees uncertainty from the mean using 95\% confidence intervals (CIs). Nano-dose and micro-dose showed the same intraoperator repeatability, whereas nano-dose was 1 degree less reproducible than micro-dose. Figures 2 and 3 illustrate Bland-Altman plots for the 2 imaging modalities, showing that measurement uncertainty did not increase with curve severity and that no systematic bias was observed.

The proportional dose reduction from micro-dose to nano-dose was $83 \%$ (ie, nano-dose was 6 times less irradiating than micro-dose). DAP values using the same collimation displayed by the EOS system have been listed in Table 1. Effective dose with the pediatric phantom in PAL exposure was $3.8 \mu \mathrm{Sv}$ (95\% CI: 3.5-4.2), whereas, previously, an effective dose of $22 \mu \mathrm{Sv}$ (95\% CI: 20-23) was reported for the same phantom in PAL projection using EOS micro-dose protocol and $157 \mu \mathrm{Sv}$ for standarddose. $^{11}$

TABLE 2. Reproducibility of Cobb Angle Measurements

\begin{tabular}{lcc}
\hline Acquisition Protocol & Micro-Dose & Nano-Dose \\
\hline Intraoperator reproducibility (deg.)* & 3.0 & 3.4 \\
Interoperator reproducibility (deg.) & 4.1 & 5.0 \\
\hline
\end{tabular}

*Reproducibility values are expressed as a variation from the mean (2 SD). 


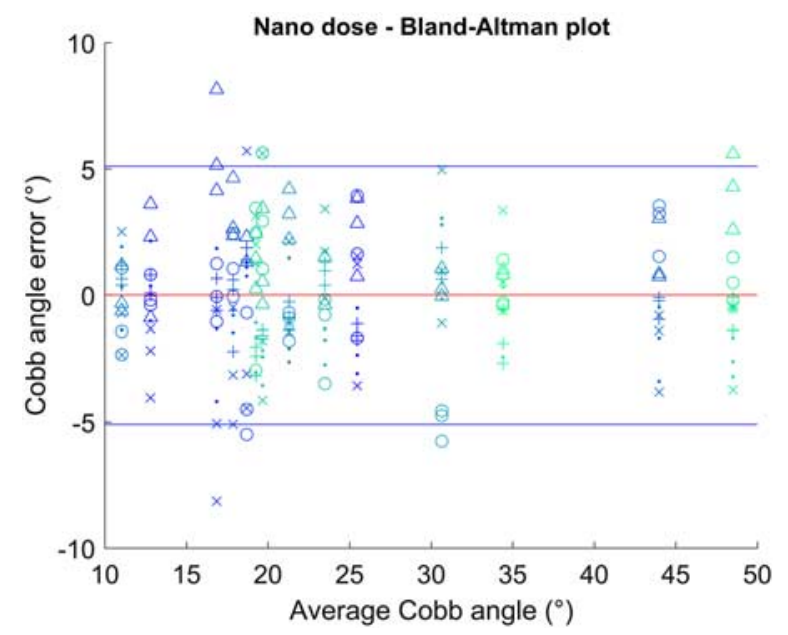

FIGURE 2. Bland-Altman plot for Cobb angle in nano-dose $\mathrm{x}$-rays. Symbols represent different operators.

\section{DISCUSSION}

To the best of our knowledge, the proposed nanodose protocol of this study offers the lowest possible dose, with existing low-dose systems allowing for reproducible Cobb angle measurements. Twenty-one consecutive patients were assessed with micro-dose and nano-dose $\mathrm{x}$-rays. Repeatability and reproducibility were good and comparable between the 2 modalities. The interobserver and intraobserver variability for both micro-dose and nano-dose was well within previously published measures for conventional radiology. $.4,15,17$

Radiation exposure to patients with the new nanodose protocol was equal to an effective dose of $\sim 3.8 \mu \mathrm{Sv}$, that is, one sixth of the existing micro-dose protocol and roughly 40 times less than the EOS standard-dose proto$\mathrm{col}$, a dose that is $<1$ day of natural background exposure - that is, the worldwide mean weekly exposure is estimated at $\sim 46 \mu \mathrm{Sv} .{ }^{19}$ This protocol values safety by means

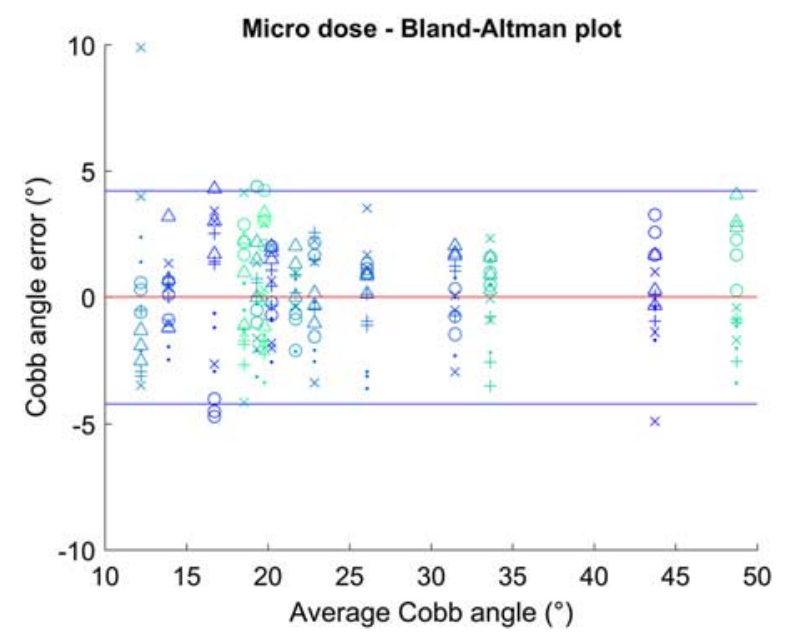

FIGURE 3. Bland-Altman plot for Cobb angle in micro-dose $\mathrm{x}$-rays. Symbols represent different operators. of lowest possible risk of stochastic health effects from ionizing radiation and adheres to the ALARA principle by still allowing for reproducible Cobb angles.

\section{Limitations of this Study}

The quality was as expected-worse for nano-dose images. Figure 4 shows micro-dose and nano-dose films. For some, it was difficult to identify the endplates. This occurred both with micro-dose and with nano-dose techniques, although more often for nano-dose, and would be expected to affect the reproducibility of the Cobb angles negatively, as described in previous studies. Nano-dose actually seemed more accurate toward defining no scoliosis (Cobb $<10$ degrees). The reason could be that, when the endplate was not easily recognizable because of reduced image quality, observers tended to use other landmarks that remain visible, such as the pedicles, and they converge toward the same result, whereas endplate orientation is not always easily interpreted even in normal quality images.

Another limitation is the fact that repeated measurements were made on preselected end vertebrae, thus omitting variable observer identification of end vertebrae. This approach was chosen to limit the source of the uncertainty to the imaging. Moreover, lateral images were not analyzed; initial qualitative analysis of these images suggest that nano-dose should solely be used for anteroposterior or posteroanterior projection in assessing the possible progression of scoliosis in the coronal plane. Although the endplate remains visible in very mild scoliosis, the interpretation of the sagittal plane in more severe scoliosis could be questionable. Regardless of image quality, it will always be less difficult to measure Cobb angle in the coronal plane in patients with hypokyphosis or normal degrees of kyphosis, as opposed to patients with hyperkyphosis in severe scoliosis.

There is always a risk of confounding, a comparatively small number of patients might lead to falsely increased repeatability. However, the order of measurement was randomized to avoid memorization of angles, and measurements were performed by 5 observers making 3 separate measurements for each modality, decreasing the risk of falsely elevated repeatability. Having 5 observers is common practice for such a study. ${ }^{15}$

The results and conclusions of this study were based on children between 9 and 12 years of age, with a $\mathrm{BMI}<26$. Further research is necessary to determine whether such low dose could provide good image quality in younger children, who present incomplete mineralization of the spine, or older children who could have higher BMI.

\section{Cobb Angle Controversy}

An accurate and reproducible measure is crucial to diagnose, follow-up, and evaluate treatment of scoliosis. 2D Cobb angle is not an exact and precise measure, as has been discussed earlier. Although more sophisticated methods exist to obtain Cobb angle and other 3D parameters of the scoliotic trunk, they are not yet widespread, and 2D Cobb angle remains the most widely used 




FIGURE 4. Micro-dose and nano-dose spine images in frontal and lateral views.

and referenced radiologic method within deformity surgery to assess curve magnitude. ${ }^{17}$ In our daily clinical practice, Cobb angle measurement is used to assess curve progression in children and adolescents during growth. A curve magnitude progression of $>5$ degrees after 6 months is consistent with progressive scoliosis and may require orthotic treatment using a brace. Nevertheless, limiting the observation of scoliosis to the coronal plane might lead to missing important factors that might be related to progression, such as sagittal global alignment, unbiased vertebral axial rotations, rib cage deformity, etc. Moreover, lowering the dose too much would be against the ALARA principle: on the one hand, it would decrease the potential risk of future cancer development. In contrast, lower quality of the image might not allow the surgeon to detect specificities of the patient or localized alterations.

\section{Dose-Optimization Controversy}

In a recent paper by Siegel et $a 1,{ }^{20}$ the issue of dose optimization has been taken up for revision. According to the authors, ionizing radiation from medical diagnostic equipment has not been proven to cause health risks with regard to radiation-induced cancer, and might, in fact, be dangerous to patients for not being diagnosed correctly instead. They do state that eliminating all nonclinically warranted medical diagnostic procedures is important, but claim that "the attempts to lessen fictitious risk by lowering dose in clinically warranted studies is a misapplication of the principles." However, the authors have not referred to any of the studies showing an association with increased risk of cancer mortality, for example, among scoliosis patients after repeated exposure to diagnostic x-rays. ${ }^{2}$ Indeed, it is extremely difficult to prove direct relations between radiation exposure and cancer, but likewise difficult to prove the opposite.

Is there a need to reduce dose further, when the EOS micro-dose, which has previously been validated with regard to image quality for scoliosis assessment, ${ }^{21}$ is already $<1$ week of natural background radiation and in accordance with ALARA?

The gain for the patient is a reduced potential risk of radiation-induced cancer in the long term. The potential risks are as follows: bad image quality and overevaluation or underevaluation of scoliosis severity. The former can be neglected, as long as obese patients and patients with metal implants are excluded. Moreover, even if an additional micro-dose or standard-dose EOS needs to be performed, the dose delivered by the nano-dose would still be negligible. Overevaluation could rise from large errors in Cobb angle measurements; given the present results, this can be excluded as well. Underevaluation of severity can arise from neglecting aspects other than Cobb angle that characterize a progressive phenotype: altered sagittal alignment, spinal torsion, etc.

\section{CONCLUSIONS}

Results show that reproducibility for the proposed nano-dose protocol is not significantly inferior to the micro-dose protocol for coronal plane Cobb angle assessment. The new protocol is not intended to take over first diagnostic imaging or presurgery planning, but we suggest it could be used for routine follow-up of children 12 years of age or younger, being observed for possible progression of scoliosis. The parents and patients would be assured that the protocol to the best of our knowledge poses no more risk than living daily life; thus, repeating images every 3-6 months would not pose any health risks or concerns. This protocol allows for safely repeated evaluations of potential Cobb angle progression.

\section{ACKNOWLEDGMENTS}

The authors acknowledge the valuable help from University College Nordjylland (Radiografskolen), 9220 Aalborg Ø, Denmark, for providing access to TLD irradiator 
and dose reader along with technical support. They also thank Professor Hubert Ducou le Pointe at the Radiological Department at Sorbonne Université, Armand Trousseau Hospital, APHP Paris, France, for helping facilitate this study. The authors are grateful to the ParisTech BiomecAM chair program on subject-specific musculoskeletal modelling (with the support of ParisTech and Yves Cotrel Foundations, Société Générale, Proteor, and Covea).

\section{REFERENCES}

1. Levy AR, Goldberg MS, Mayo NE, et al. Reducing the lifetime risk of cancer from spinal radiographs among people with adolescent idiopathic scoliosis. Spine (Phila Pa 1976). 1996;21: $1540-1547$.

2. Doody MM, Ronckers CM, Land CE, et al. Cancer mortality among women frequently exposed to radiographic examinations for spinal disorders. Radiat Res. 2010;174:83-90.

3. Simony A, Christensen SB, Jensen KE, et al. Incidence of cancer and infertility, in patients treated for adolescent idiopathic scoliosis 25 years prior. Eur Spine J. 2015;24(suppl 1):S740.

4. The International Commission on Radiological Protection. The 2007 Recommendations of the International Commission on Radiological Protection. ICRP Publication 103. Ann ICRP. 2007;37:2-4.

5. Strauss KJ, Kaste SC. ALARA in pediatric interventional and fluoroscopic imaging: striving to keep radiation doses as low as possible during fluoroscopy of pediatric patients-a white paper executive summary. J Am Coll Radiol. 2006;3:686-688.

6. Melhem E, Assi A, El Rachkidi R, et al. EOS® biplanar X-ray imaging: concept, developments, benefits, and limitations. $J$ Child Orthop. 2016;10:1-14.

7. Deschênes S, Charron G, Beaudoin G, et al. Diagnostic imaging of spinal deformities: reducing patients radiation dose with a new slot-scanning X-ray imager. Spine (Phila Pa 1976). 2010;35: 989-994.

8. Yvert M, Diallo A, Bessou P, et al. Radiography of scoliosis: Comparative dose levels and image quality between a dynamic flatpanel detector and a slot-scanning device (EOS system). Diagn Interv Imaging. 2015;96:1177-1188.
9. Kalifa G, Charpak Y, Maccia C, et al. Evaluation of a new low-dose digital X-ray device: first dosimetric and clinical results in children. Pediatr Radiol. 1998;28:557-561.

10. Damet J, Fournier P, Monnin P, et al. Occupational and patient exposure as well as image quality for full spine examinations with the EOS imaging system. Med Phys. 2014;41:063901.

11. Pedersen PH, Peterson AG, Østgaard SE, et al. EOS® micro-dose protocol: first full-spine radiation dose measurements in anthropomorphic phantoms and comparisons with EOS standard-dose and conventional digital radiology. Spine (Phila Pa 1976). 2018;43: E1313-E1321.

12. Humbert L, De Guise JA, Aubert B, et al. 3D reconstruction of the spine from biplanar X-rays using parametric models based on transversal and longitudinal inferences. Med Eng Phys. 2009;31:681-687.

13. Lechner R, Putzer D, Dammerer D, et al. Comparison of two- and three-dimensional measurement of the Cobb angle in scoliosis. Int Orthop. 2017:41:957-962.

14. Kuklo TR, Potter BK, Schroeder TM, et al. Comparison of manual and digital measurements in adolescent idiopathic scoliosis. Spine (Phila Pa 1976). 2006;31:1240-1246.

15. Langensiepen S, Semler O, Sobottke R, et al. Measuring procedures to determine the Cobb angle in idiopathic scoliosis: a systematic review. Eur Spine J. 2013;22:2360-2371.

16. Vrtovec T, Pernuš F, Likar B. A review of methods for quantitative evaluation of spinal curvature. Eur Spine J. 2009;18:593-607.

17. Papaliodis D, Bonanni P, Roberts $\mathrm{T}$, et al. Computer assisted Cobb angle measurements: a novel algorithm. Int J Spine Surg. 2017;11:167-172.

18. Computerized Imaging Reference Systems, Inc. Whole body dose, organ dose, therapeutic dose. ATOM Dosimetry Phantoms; 2013. Available at: http://www.cirsinc.com/wp-content/uploads/2019/05/701706-ATOM-PB-120418.pdf. Accessed May 24, 2019.

19. United Nations Scientific Committee on the Effects of Atomic Radiation (UNSCEAR). Sources and effects of ionizing radiation; 2010.

20. Siegel JA, Sacks B, Pennington CW, et al. Dose optimization to minimize radiation risk for children undergoing $\mathrm{CT}$ and nuclear medicine imaging is misguided and detrimental. $J$ Nucl Med. 2017;58:865-868.

21. Ilharreborde B, Ferrero E, Alison M, et al. EOS microdose protocol for the radiological follow-up of adolescent idiopathic scoliosis. Eur Spine J. 2016;25:526-531. 